\title{
Research Article \\ Study of the Protection of Aluminum Alloy Surfaces by a Graphene-Modified Fluorocarbon Anticorrosive Coating
}

\author{
Peng Wang $(\mathbb{D}$ and Dayong Cai \\ College of Materials Science and Engineering, Yanshan University, Qinhuangdao 066004, China \\ Correspondence should be addressed to Peng Wang; lrwp@ysu.edu.cn
}

Received 15 August 2020; Revised 18 September 2020; Accepted 5 October 2020; Published 23 October 2020

Academic Editor: Yimin Wu

Copyright ( 2020 Peng Wang and Dayong Cai. This is an open access article distributed under the Creative Commons Attribution License, which permits unrestricted use, distribution, and reproduction in any medium, provided the original work is properly cited.

Graphene-modified anticorrosion coatings have become a hot spot in the field of metal protection due to the large-scale promotion of aluminum alloys, which are prone to corrosion in marine and atmospheric environments. The protection of aluminum alloy surfaces by a graphene-modified anticorrosive coating was explored in this study by applying a graphene-modified anticorrosive coating to an aluminum alloy surface to test its resistance to corrosion. Dispersion-treated reduced graphene oxide (rGO) was used to modify the epoxy resin and fluorocarbon resin. It was found, by using a scanning electron microscopy (SEM) and the microstructure of the coating made by the Raman Spectroscopy Institute, that the addition of rGO could effectively improve the porosity of the epoxy primer, and the electrochemical workstation was able to resist the graphene-modified anticorrosive coating. The corrosion performance was quickly characterized, the polarization curve and the AC impedance curve were fitted, and it was found that the self-corrosion current density $\left(J_{\text {corr }}\right)$ of the graphene-modified anticorrosive coating was the smallest $\left(1.190 \times 10^{-7} \mathrm{~A} / \mathrm{cm}^{2}\right)$ when $0.6 \%$ of $\mathrm{rGO}$ was added; the impedance modulus $(|Z|)$ was the largest $\left(10^{4}\right)$, the capacitive reactance arc radius was the largest, and the coating resistance was the largest after fitting (15517 $\Omega$ ). When $0.8 \%$ of rGO was added, the dispersion coefficient was large, and it had a good physical insulation performance. The main reason for the reduction of the corrosion resistance was that the agglomeration of rGO made the aluminum alloy matrix and the external corrosive environment form a highly conductive circuit, thereby accelerating the corrosion of the aluminum alloy matrix.

\section{Introduction}

In a space shuttle, the most serious corrosion is found in the structure of the aircraft body. A large amount of condensation forms on the surface and inside of the aircraft fuselage during the takeoff and climb phase due to the difference in temperature between the ground and the high altitude. When the relative humidity of the atmosphere in the external environment exceeds $65 \%$, a film of water about $0.001 \mu \mathrm{m}$ thick is deposited on the surface of the aircraft body and structural parts [1]. The thickness of the water film gradually increases with the increase of the external relative humidity, and when the humidity of the external environment reaches $100 \%$, condensation forms on the surface of the aircraft body. Corrosion is caused by the saturated $\mathrm{Cl}^{-}$ion, $\mathrm{O}_{2}$, and other corrosive media coming into contact with the aluminum alloy structural parts of the aircraft. The common types of corrosion of the aluminum alloy structural parts of aircraft currently include the following: (1) pit-like corrosion: in a neutral aqueous solution or humid environment, corrosion pits appear on the surface of the aluminum alloy. Their depth and diameter increase rapidly, and they continue to develop inside the aluminum alloy. In a marine atmosphere, $\mathrm{Cl}^{-}$ions can accelerate the corrosion of aluminum alloy, and the surface of aluminum alloy is prone to a galvanic effect; (2) crevice corrosion: the aluminum alloy undergoes a reduced reaction of anode solvent and cathode oxygen in the gap between the interface, resulting in an accumulation of corrosion products, blocking the transmission of corrosive media, leading to differences in the corrosive media and concentration inside and outside the crevice, and then evolving into "occluded battery corrosion"; and (3) friction corrosion: corrosion products are formed by chemical and electrochemical reactions on the contact surfaces of two aluminum alloy 
structural parts. The friction between the contact surfaces causes the corrosion products to fall off, and the newly exposed aluminum alloy surface corrodes again and again, which causes damage to the aluminum alloy structural parts. Organic coating protection is the simplest, most efficient, and economical anticorrosion method in the industry [2].

The level of development of heavy-duty anticorrosion coatings is a measure of the advanced level of a country's coatings industry. A series of heavy-duty anticorrosion coatings have been developed at home and abroad in response to the phenomenon that aluminum alloys are prone to corrosion. These mainly include the following: (1) epoxy heavyduty anticorrosion coatings, an epoxy resin macromolecule chain, which contains two or more epoxy groups. Thermosetting resin is highly adhesive and extremely hard. As such, it is widely used for building materials. However, epoxy resin is extremely porous, It is easily corroded by oxygen, water, and chloride ions and has poor acid resistance. Other disadvantages include poor ultraviolet rays and brittle paint film; (2) polyurethane heavy-duty anticorrosive coating; this is extremely flexible and highly adhesive and has excellent wear resistance due to the reactive cyanate group (-NCO) on the macromolecular chain. It is often used as a topcoat in the field of heavy anticorrosion to protect the substrate due to its mechanical properties, but its UV resistance is poor, and its anticorrosion performance is greatly reduced after ultraviolet aging; (3) fluorocarbon coatings that contain a large amount of $\mathrm{C}$, with a bond energy of up to $485.6 \mathrm{~kJ} / \mathrm{mol}$ F-bonded fluorocarbon resin, have excellent chemical and UV resistance. They are widely used in ships, pipelines, and aerospace fields, but their adhesion and pigment wetting are poor $[3,4]$.

These reasons have led to much research by scientific researchers. For example, Pourhashem and others [5] used silane coupling agents APTES (KH550) and GPTMS (KH560) to prepare graphene oxide-modified epoxy resin coatings to test their ability to protect stainless steel. Ye et al. [6] used covalent grafting to prepare functionalized graphene-modified epoxy resin coating and explored the corrosion resistance of Q235 steel; Uzoma et al. [7] used a solution polymerization of acrylic monomer to synthesize hydrophobic organic. The siloxane-acrylic resin and fluorosilane-modified graphene nanosheets were connected by covalent bonds to prepare a superhydrophobic $\left(\geq 152^{\circ}\right)$ anticorrosion coating, which improved the protection of LY12 aluminum alloy. In the above research on graphenemodified anticorrosion coatings, graphene oxide $(\mathrm{GO})$ was used to modify anticorrosion coatings, the protection mechanism of stainless steel was explored, and the modified anticorrosion coatings of reduced graphene oxide were used on aviation aluminum alloy substrates $[8,9]$. Therefore, due to the limited exploration, the contents defined below are addressed in this article.

A polymer dispersant is used to produce an $\mathrm{rGO}$ surface to prepare for graphene dispersion; then, rGO is used to modify the epoxy primer and fluorocarbon resin topcoat; and atmospheric spray technology is used to apply a graphene-modified anticorrosive coating to the surface of aviation aluminum alloy. An electron microscope and
Raman spectroscopy are used to scan, test, and characterize the microstructure of the graphene-modified anticorrosive coating; and an electrochemical workstation is used to test and characterize the corrosion resistance of the graphenemodified anticorrosive coating. The corrosion resistance mechanism of the anticorrosive coating is explored by fitting an equivalent circuit to explore its ability to resist the corrosion of aviation aluminum alloy.

\section{Experimental Section}

2.1. Materials. The metal matrix used in the experiment is 7075-T6 aluminum alloy, and its composition is shown in Table 1. The coating is epoxy resin primer and fluorocarbon resin topcoat provided by the Beijing Institute of Aeronautical Materials. The reduced graphene oxide is 1133 anticorrosive graphene produced by the China Changzhou Sixth Element Material Technology Co., Ltd. The graphene dispersant used is F431 type dispersant produced by the Foshan Aona Polymer Co., Ltd. The other chemical reagents used throughout the experiment were purchased from Aladdin.

2.2. Preparation of Anticorrosion Coatings. Firstly, the reduced graphene oxide, F431 dispersant, and xylene solution were mixed in a ratio of $1: 0.1: 10$ and then ultrasonically treated in a $600 \mathrm{~W}$ water bath sonicator for 2 hours to obtain a uniform graphene dispersion. Before being painted, the surface of the 7075-T6 aluminum alloy was sandblasted using 120-mesh white corundum. Then, it was ultrasonically degreased with acetone, dehydrated with ethanol, and finally dried and placed in a desiccator for use. The epoxy resin, curing agent, and diluent were mixed at $8: 1: 3$. After mixing the paint, the prepared graphene dispersion ( $\mathrm{rGO}$ addition ratio was $0.4 \% \mathrm{wt}$ ) was doped with reduced graphene oxide. The epoxy resin was magnetically stirred for 20 minutes using atmospheric spraying technology, sprayed on the sandblasted aluminum alloy surface, and cured at $70^{\circ} \mathrm{C}$ for 1 hour (air pressure was maintained at 6-7 MPa during spraying, and the spraying distance was maintained at $15 \sim 20 \mathrm{~cm}$ ). When the epoxy resin coating was completely dry, the above steps were repeated and proportions were added to prepare a polyurethane resin coating on the surface of the epoxy primer. The total thickness of the anticorrosive coating was about $150 \mu \mathrm{m}$. The anticorrosive coating doped with nonreduced graphene oxide was prepared in the same way as a blank comparison reference.

2.3. Characterization. A scanning electron microscope (7900F) was used to observe the microscopic morphology of the coating cross-section; a Raman spectrometer (wavelength $532 \mathrm{~nm}$ ) was used to measure the Raman spectrum of the coating to study the microstructure of the reduced graphene in the coating; an Autolab PGSTAT 302N electrochemical workstation (Switzerland Wantong) was used to determine the polarization curve and the AC impedance of the anticorrosion coating to analyze the corrosion resistance of the anticorrosion coating and the change of the law of the corrosion resistance of the graphene-modified anticorrosion coating after being soaked in $3.5 \% \mathrm{NaCl}$. NOVA software 
TABLE 1: 7075-T6 aluminum alloy composition.

\begin{tabular}{lccccccccccc}
\hline Composition & $\mathrm{Zn}$ & $\mathrm{Mg}$ & $\mathrm{Cu}$ & $\mathrm{Si}$ & $\mathrm{Fe}$ & $\mathrm{Mn}$ & $\mathrm{Cr}$ & $\mathrm{Ti}$ & $\mathrm{Al}$ & $\mathrm{Else}$ \\
\hline Percentage (\%) & 5.6 & 2.5 & 1.4 & 0.35 & 0.42 & 0.29 & 0.22 & 0.1 & $\mathrm{Margin}$ & 0.15 \\
\hline
\end{tabular}

was used to fit the electrochemical curve to draw the equivalent circuit. It had to be connected to the copper wire and then sealed with epoxy resin before being tested. The exposed coating area was fixed to $1 \mathrm{~cm}^{2}$. The electrochemical measurement selected a three-electrode system: $10 \times 10 \mathrm{~mm}$. The platinum sheet was the counter electrode, the saturated calomel electrode was the reference electrode, the polarization curve test interval was relative to the self-corrosion point $\pm 150 \mathrm{mV}$, the scanning speed was $1 \mathrm{mV} / \mathrm{s}$, the $\mathrm{AC}$ impedance test (EIS) sine wave frequency range was $0.01 \mathrm{~Hz} \sim 100000 \mathrm{~Hz}$, and the load AC disturbance voltage amplitude was $10 \mathrm{mV}$.

\section{Results and Discussion}

3.1. Morphology of rGO in Anticorrosion Coatings. There are three characteristic peaks in the Raman spectrum of melene: the $D$ peak (near $1350 \mathrm{~cm}^{-1}$ ), the $G$ peak (near $1580 \mathrm{~cm}^{-1}$ ), and the $2 \mathrm{D}$ peak (near $2690 \mathrm{~cm}^{-1}$ ). Peak D is a defect peak and requires at least one defect to activate; the $2 \mathrm{D}$ peak of a single-layer graphene is located near $2690 \mathrm{~cm}^{-1}$. It is sharp, and its intensity is about 4 times that of peak G. As the number of graphene layers increases, its position will shift to the right. The peak intensity decreases, and the peak width grows. When the graphene sheet reaches more than ten layers, its $2 \mathrm{D}$ peak shape is basically the same as graphite, but the 2D peak of graphite is composed of two peaks, 2D1 and 2D2, and the peak intensity is about $1 / 4$ and $1 / 2$ of the G peak [10].

F431 is a dispersant, and the Raman spectrum of the graphene-modified anticorrosive coating with $0.2 \%$ rGO addition is shown in Figure 1. It can be seen from Figure 1 that the intensity of the $\mathrm{D}$ peak is higher, indicating more rGO edges and defects; I2D:IG is $1: 1$, indicating that the dopant is rGO with more layers instead of graphite. This is due to the strong van der Waals force on the surface of rGO that makes rGO agglomerate inside the coating, which is consistent with the phenomenon observed by the scanning electron microscopy [11].

3.2. Morphology of Anticorrosion Coatings. Figures 2(a)-2(d) show the microscopic cross-sectional morphology of graphene-modified anticorrosion coatings with rGO additions of $0.2 \%, 0.4 \%, 0.6 \%$, and $0.8 \%$. It can be seen from Figures $2(\mathrm{a})-2(\mathrm{c})$ that there are fewer pores in the crosssection of the epoxy primer when less than 0.6 of $\mathrm{rGO}$ is added, which indicates that a small amount of rGO can effectively improve the pores in the epoxy primer. As the corrosion progresses, it can effectively prevent the intrusion of water molecules, chloride ions, and other corrosive media; reduce the accumulation of corrosive ions; greatly reduce the amount of "water" in the graphene-modified anticorrosive coating; and increase the probability of forming an "air

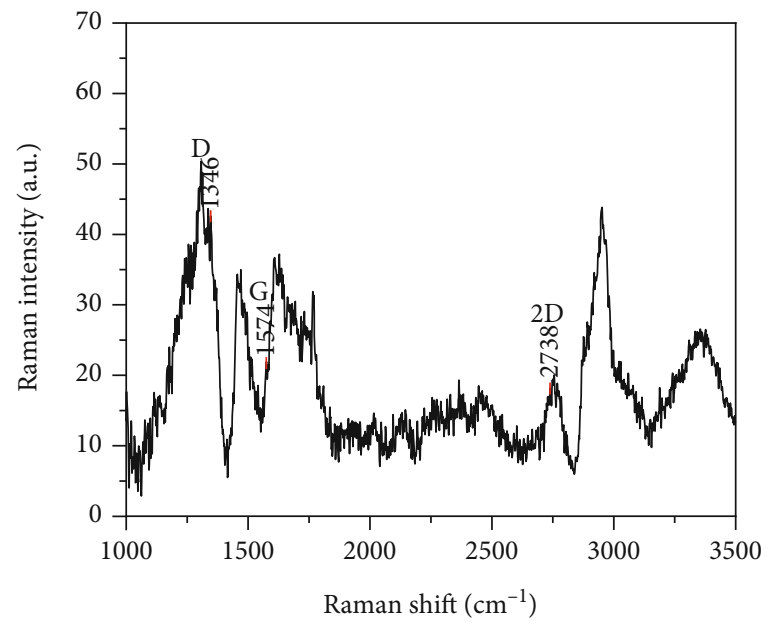

FIGURE 1: Raman of graphene-modified coatings.

passage"; and the pores in the epoxy primer cross-section increase sharply with $0.8 \%$ of $\mathrm{rGO}[12,13]$. As the corrosion progresses, the corrosive media accumulate in the pores, reducing the graphene-modified anticorrosive coating. The corrosion resistance of the layer accelerates the corrosion of the aluminum alloy substrate, and the dispersion of rGO in the cross-section of the polyurethane resin topcoat in each graphene-modified anticorrosive coating is relatively uniform. No microcracks appear as the amount of rGO is increased. The rGO in the anticorrosive coating can accelerate the electron transfer speed in the corrosion process, increase the conduction path of electrons, and effectively utilize the anticorrosive filler in the polyurethane resin topcoat $[14,15]$.

The statistical results of the porosity of the graphenemodified anticorrosive coatings with $0.2 \%, 0.4 \%, 0.6$, and $0.8 \%$ of the coatings in the prepared state and with F431 as the dispersant and $0.8 \%$, respectively, are shown in Table 2 using Image-Pro software. It can be seen that the porosity of the primer coating tended to first decrease and then increase when less than $0.6 \%$ of $\mathrm{rGO}$ was added. The porosity of the primer coating was smallest at $0.12 \%$ when $0.6 \%$ of rGO was added, and the topcoat was defective when $0.8 \%$ of rGO was added [16]. This shows that the dispersion of rGO in the coating affects its microscopic morphology. The better the dispersion effect of rGO, the greater the effect of its microlamellar structure. The addition of an excessive amount of rGO will cause the rGO agglomeration phenomenon to continue to increase until the graphene-modified anticorrosion coating becomes defective, thereby reducing its ability to resist corrosion [17].

3.3. Electroactivity of Anticorrosion Coatings. The polarization curves of the prepared state and F431 as a dispersant with $\mathrm{rGO}$ at $0.2 \%, 0.4 \%, 0.6 \%$, and $0.8 \%$ graphene-modified 


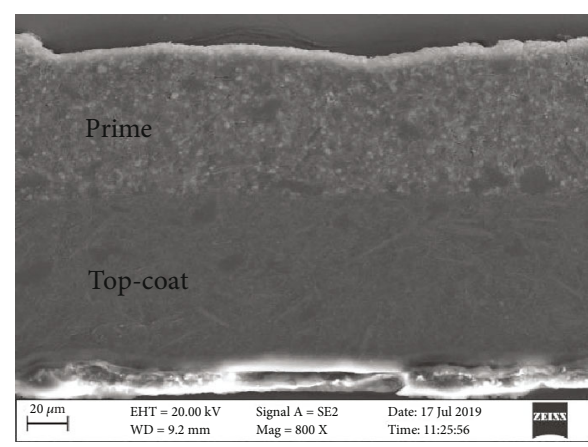

(a)

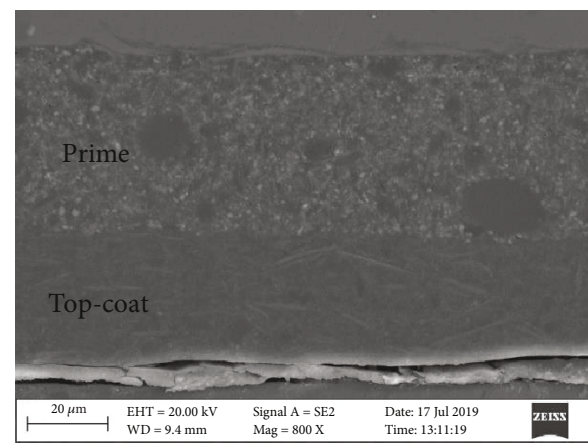

(c)

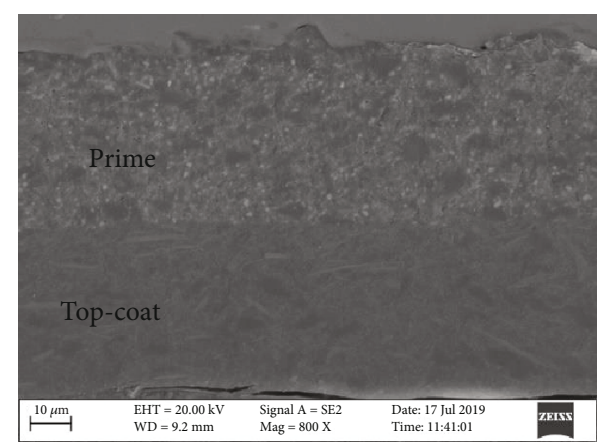

(b)

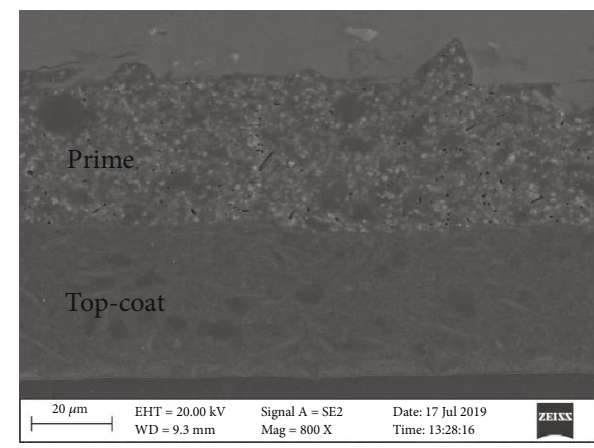

(d)

FIGURE 2: SEM of graphite-modified anticorrosive coatings with different percentages of rGO dispersed by F431 (a-d); the additional rGO are $0.2 \%, 0.4 \%, 0.6 \%$, and $0.8 \%$.

TABLE 2: Porosity of original and graphene-modified coatings.

\begin{tabular}{lcccc}
\hline Coatings & rGO-0.2\% & rGO-0.4\% & rGO-0.6\% & rGO-0.8\% \\
\hline Porosity (\%) & 0.18 & 0.12 & 0.05 & 0.63 \\
\hline
\end{tabular}

anticorrosive coating are shown in Figure 3. The size of the self-corrosion potential shows the degree of difficulty of its corrosion, and the self-corrosion current density is an important basis for characterizing its resistance to corrosion [18]. It can be seen from Figure 3 that the self-corrosion potential of the coating first appears with an increase in the amount of rGO. After the movement, the negative trend reached the most positive value when the amount of rGO was $0.6 \%$, which was $-0.19 \mathrm{~V}$. Then, the corrosion potential quickly became negative at $-0.65 \mathrm{~V}$ with the increase in the amount of rGO. The excellent conductivity of rGO accelerated the circulation of the electrons, increased the conduction path of the electrons inside the coating, and changed the oxide film on the aluminum alloy surface during the polarization process, which hindered the anode reaction to varying degrees (dissolution of the aluminum alloy matrix) and the cathodic reaction (oxygen absorption corrosion), leading to changes in the anode Tafel slope and the cathode Tafel slope, resulting in a positive or negative shift of the potential of selfcorrosion $[19,20]$.

The results of the Tafel curve fitting using Nova software are shown in Table 3, where $b_{\mathrm{a}}$ was the Tafel slope of the anode, $b_{\mathrm{c}}$ was the Tafel slope of the cathode, and $R_{\mathrm{p}}$ was the polarization resistance calculated based on a ratio of 3.1. It can be seen from the fitting data in Table 3 that the

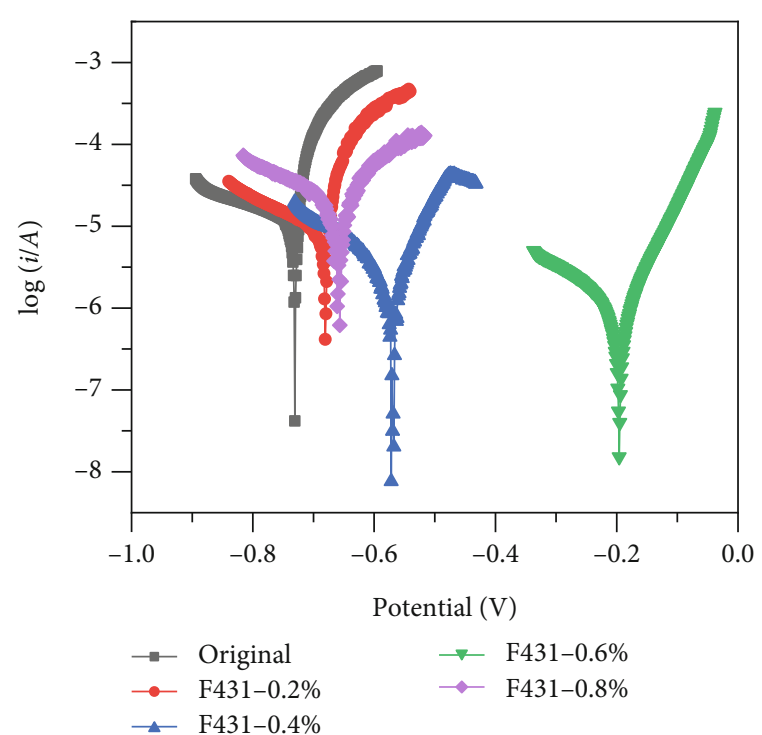

Figure 3: Tafel curves of original and graphene additions of $0.2 \%$, $0.4 \%, 0.6 \%$, and $0.8 \%$ anticorrosion coatings. Original, no graphene added.

coating self-corrosion current density decreased and the polarization resistance value increased when the addition of rGO increased. The self-corrosion current density $\left(J_{\text {corr }}\right)$ reaches the minimum when $0.6 \%$ of $\mathrm{rGO}$ was added, the value was $1.19 \times 10^{-7} \mathrm{~A} / \mathrm{cm}^{2}$, and the polarization resistance was the largest at $29831 \Omega$. The self-corrosion current density 
TABLE 3: Results of Tafel curve fitting.

\begin{tabular}{lccccc}
\hline & $E_{\text {corr }}(\mathrm{V})$ & $J_{\text {corr }}\left(\mathrm{A} / \mathrm{cm}^{2}\right)$ & $b_{\mathrm{a}}$ & $b_{\mathrm{c}}$ & $R_{\mathrm{p}}(\Omega)$ \\
\hline Original & -0.7313 & $2.147 \times 10^{-6}$ & 0.012724 & 0.007797 & 977.8 \\
F431-0.2\% & -0.6805 & $1.580 \times 10^{-6}$ & 0.008116 & 0.009737 & 1220.8 \\
F431-0.4\% & -0.56789 & $4.633 \times 10^{-7}$ & 0.017767 & 0.033082 & 10836 \\
F431-0.6\% & -0.1956 & $1.190 \times 10^{-7}$ & 0.014154 & 0.019352 & 29831 \\
F431-0.8\% & -0.6592 & $1.758 \times 10^{-6}$ & 0.013298 & 0.013453 & 1651.9 \\
\hline
\end{tabular}

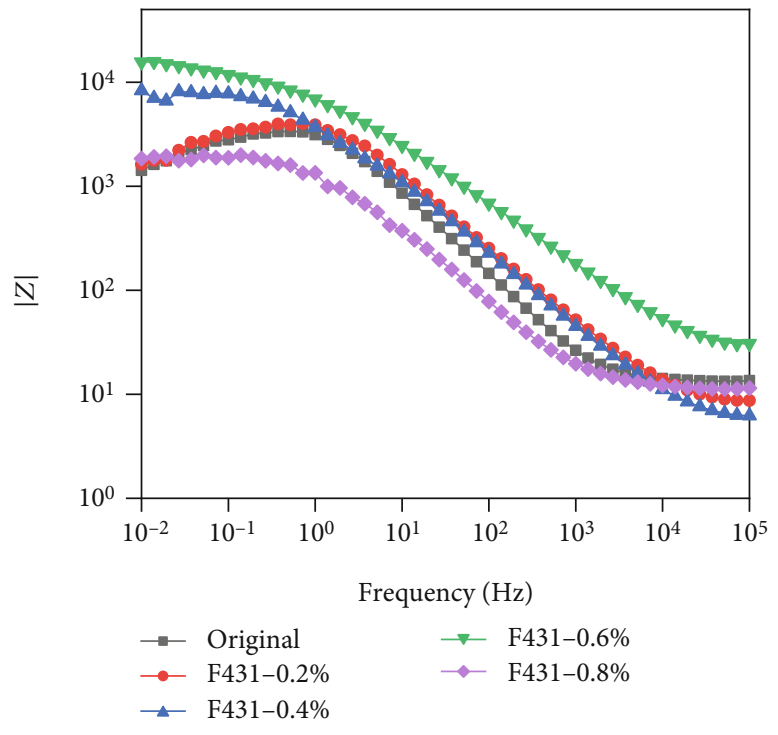

(a)

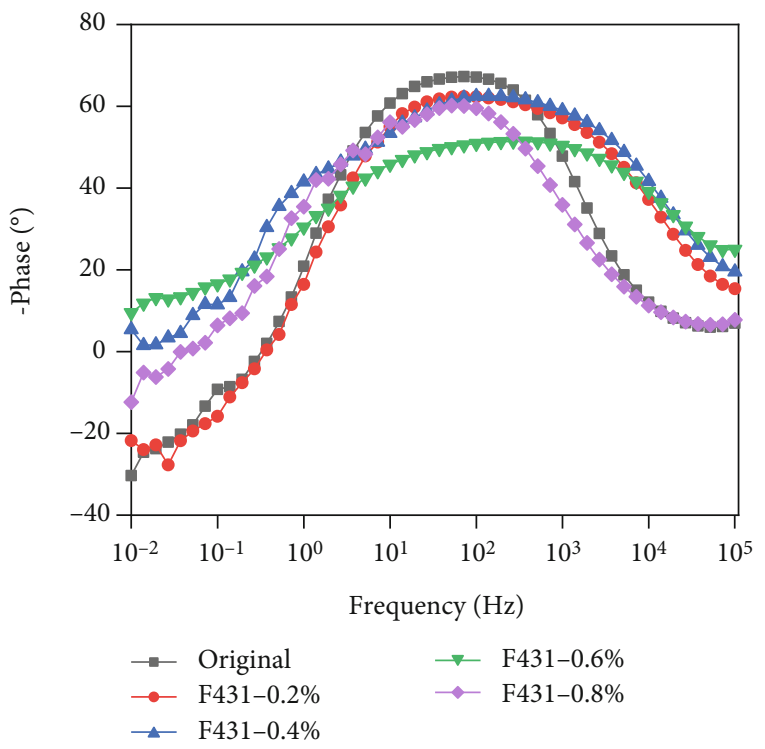

(b)

Figure 4: Bode of original and graphene additions of $0.2 \%, 0.4 \%, 0.6 \%$, and $0.8 \%$ to anticorrosion coatings.

$\left(J_{\text {corr }}\right)$ increased to $1.75 \times 10^{-6} \mathrm{~A} / \mathrm{cm}^{2}$ when $0.8 \%$ of $\mathrm{rGO}$ was added, the polarization resistance value decreased to 1657.9 $\Omega$, and the corrosion resistance of the graphenemodified anticorrosion coating dropped sharply. This is because the addition of rGO increases the conduction path of the electrons in the anticorrosion coating, thereby increasing its corrosion resistance performance. When $0.8 \%$ of $\mathrm{rGO}$ is added, it will accumulate in the coating to form a conductive path to connect to the external corrosive environment and accelerate the exchange of electrons between the aluminum alloy surface and the metal ions in the corrosive environment, thereby reducing the corrosion resistance of the coating performance $[20,21]$.

$$
R_{\mathrm{p}}=\frac{b_{\mathrm{a}} / b_{\mathrm{c}}}{2.303\left(b_{\mathrm{a}}+b_{\mathrm{c}}\right) J_{\text {corr }}} .
$$

The Bode curves of the prepared state and F431 as a dispersant with the addition of $\mathrm{rGO}$ at $0.2 \%, 0.4 \%, 0.6 \%$, and $0.8 \%$ to the graphene-modified anticorrosive coating are shown in Figure 4. It can be seen from Figure 4(a) that the impedance modulus value of the graphene-modified anti- corrosive coating is at low frequency when F447 is used as the dispersant and the amount of rGO added is increased from $0.2 \%$ to $0.8 \%$. $|Z|$ shows a tendency to increase first and then decrease. When the amount of rGO added is $0.6 \%$, the impedance modulus value at low frequency is the largest, about 105, indicating that the corrosion resistance of the anticorrosive coating is the best at this time, and the polarization curve results are consistent $[22,23]$.

The prepared state and F431 as a dispersant and the Nyquist curve of the graphene-modified anticorrosion coating with $0.2 \%, 0.4 \%, 0.6 \%$, and $0.8 \%$ of added rGO are shown in Figure 5, and the equivalent circuit was analyzed using Nova software. The middle components are fitted (Figure 6(a) is the equivalent circuit diagram of the prepared state when the amount of $\mathrm{rGO}$ is $0.2 \%$ and $0.8 \%$, and Figure $6(\mathrm{~b})$ is the equivalent circuit diagram when the amount of $\mathrm{rGO}$ is $0.4 \%$ and $0.6 \%$ ); $R_{\mathrm{s}}$ is the solution resistance; $Q_{\mathrm{dl}}$ is the constant phase angle element; $R_{\mathrm{ct}}$ is the coating resistance; $L$ is the inductance caused by pitting corrosion; $R_{\mathrm{m}}$ is the oxide film resistance, and the equivalent circuit component values are shown in Table 4 . It can be seen by combining the AC impedance spectroscopy with the fitted coating resistance 


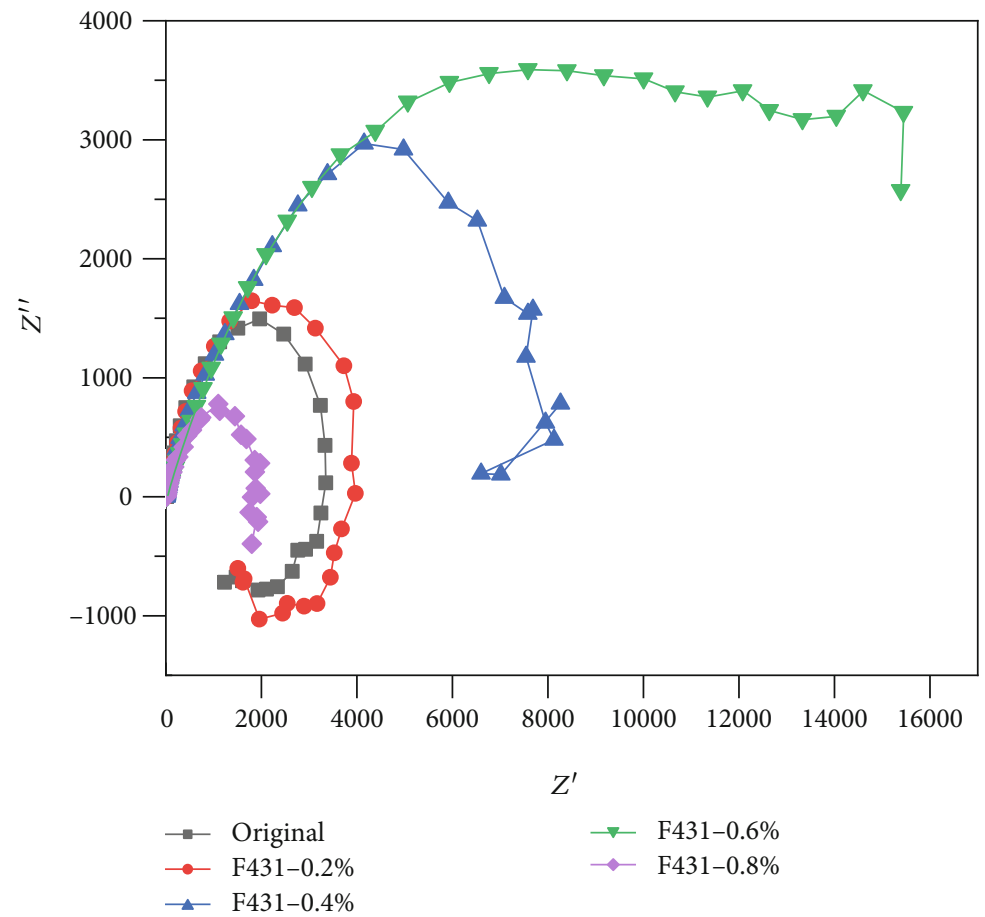

FIGURE 5: Nyquist of original and graphene additions of $0.2 \%, 0.4 \%, 0.6 \%$, and $0.8 \%$ anticorrosion coatings. Original, no added graphene; (1-4) $0.2 \%, 0.4 \%, 0.6 \%$, and $0.8 \%$ added graphene.

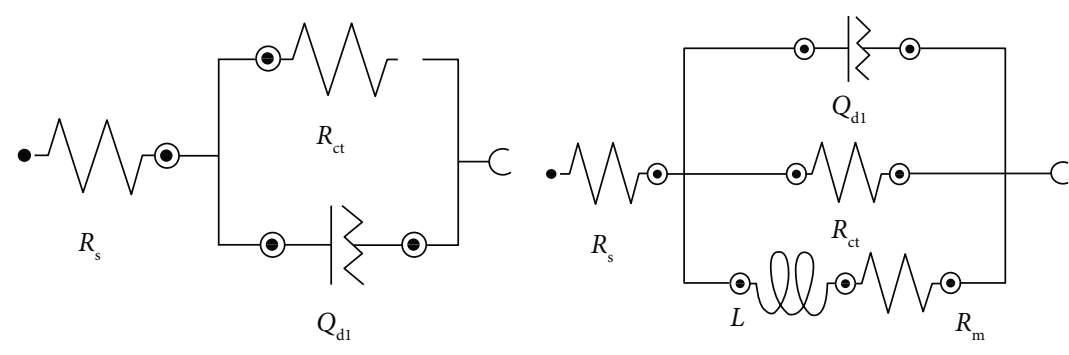

(a)

(b)

Figure 6: Equivalent circuit diagram.

TABLE 4: Fitting data of equivalent circuit components.

\begin{tabular}{lcccc}
\hline Coatings & $R_{\mathrm{s}}(\Omega)$ & $R_{\mathrm{ct}}(\Omega)$ & $Y_{0}$ & $N$ \\
\hline Original & 13.372 & 2230.4 & $2.614 \times 10^{-5}$ & 0.870 \\
F431-0.2\% & 8.072 & 2532.1 & $2.410 \times 10^{-5}$ & 0.776 \\
F431-0.4\% & 4.749 & 8177.1 & $4.857 \times 10^{-5}$ & 0.704 \\
F431-0.6\% & 17.863 & 15517.0 & $3.212 \times 10^{-5}$ & 0.595 \\
F431-0.8\% & 10.944 & 1957.7 & $1.147 \times 10^{-4}$ & 0.744 \\
\hline
\end{tabular}

data that F431 is a dispersant, and the capacitive arc radius in the Nyquist curve first increases and then decreases when the amount of $\mathrm{rGO}$ is increased from $0.2 \%$ to $0.8 \%$. The capacitive arc radius is the largest when the amount of $\mathrm{rGO}$ is $0.6 \%$. At this time, the resistance of the graphene-modified anticorrosion coating is $15517 \Omega$, and the graphene-modified anticorrosion coating has better corrosion resistance, which is consistent with the polarization curve. The inductive arc resistance appears in the Nyquist curve in the preparation state and the addition of $\mathrm{rGO}$ at $0.2 \%$ and $0.8 \%$, indicating that the corrosion ion $\mathrm{Cl}^{-}$has appeared on the surface of the oxide film of the aluminum alloy substrate $[24,25]$.

\section{Conclusions}

(1) The excellent microsheet structure of graphene improves defective anticorrosive coating. The porosity of the graphene-modified anticorrosive coating is the smallest when $0.6 \%$ of rGO is added. The agglomeration of rGO becomes serious as more is added, and rGO with low surface energy cannot be fully mixed with organic coatings; therefore, new defects will occur after the coating has been cured 
(2) The self-corrosion current density $\left(J_{\text {corr }}\right)$ of the graphene-modified anticorrosive coating is the smallest $\left(1.190 \times 10^{-7} \mathrm{~A} / \mathrm{cm}^{2}\right)$ when $0.06 \%$ of $\mathrm{rGO}$ is added, and the impedance modulus $(|Z|)$ is the largest $\left(10^{4}\right)$. In terms of the capacitive reactance, the arc radius is the largest, and the coating resistance is the largest $(15517 \Omega)$ after fitting. The corrosion resistance is the best at this time, mainly because the graphene is doped into the coating, extending the conduction path of electrons inside the coating, increasing the resistance of the coating, and decelerating corrosion

(3) The rGO agglomeration phenomenon in the graphene-modified anticorrosive coating is serious when $0.8 \%$ of rGO is added, the coating defects increase, the self-corrosion current density $\left(J_{\text {corr }}\right)$ is larger $\left(1.758 \times 10^{-6} \mathrm{~A} / \mathrm{cm}^{2}\right)$, and the coating is resistant. The corrosion performance is poor, but its dispersion coefficient is large, and it has a good physical insulation performance. Its corrosion resistance is reduced mainly because the agglomeration of rGO makes the aluminum alloy matrix, and the external corrosive environment forms a highly conductive circuit, thereby accelerating the corrosion of the aluminum alloy matrix

\section{Data Availability}

The data used to support the findings of this study are available from the corresponding author upon request.

\section{Conflicts of Interest}

The authors declare no conflict of interest.

\section{References}

[1] V. K. Beura, C. Kale, S. Srinivasan, C. L. Williams, and K. N. Solanki, "Corrosion behavior of a dynamically deformed AlMg alloy," Electrochimica Acta, vol. 354, article 136695, 2020.

[2] H. Zhu, L. Yue, C. Zhuang et al., "Fabrication and characterization of self-assembled graphene oxide/silane coatings for corrosion resistance," Surface \& Coatings Technology, vol. 304, pp. 76-84, 2016.

[3] J. Mondal, L. Aarik, J. Kozlova et al., "Functionalization of Titanium Alloy Surface by graphene and Metal Oxides: Corrosion Inhibition," Journal of Nanoscience and Nanotechnology, vol. 15, no. 9, pp. 6533-6540, 2015.

[4] B. Zhang, V. R. Patlolla, D. Chiao, D. K. Kalla, H. Misak, and R. Asmatulu, "Galvanic corrosion of $\mathrm{Al} / \mathrm{Cu}$ meshes with carbon fibers and graphene and ITO-based nanocomposite coatings as alternative approaches for lightning strikes," The International Journal of Advanced Manufacturing Technology, vol. 67, no. 5-8, pp. 1317-1323, 2013.

[5] S. Pourhashem, A. Rashidi, M. R. Vaezi, and M. R. Bagherzadeh, "Excellent corrosion protection performance of epoxy composite coatings filled with amino-silane functionalized graphene oxide," Surface \& Coatings Technology, vol. 317, pp. 1-9, 2017.
[6] Y. Ye, D. Zhang, T. Liu et al., "Superior corrosion resistance and self-healable epoxy coating pigmented with silanzied trianiline-intercalated graphene," Carbon, vol. 142, pp. 164176, 2019.

[7] P. C. Uzoma, F. Liu, L. Xu et al., "Superhydrophobicity, conductivity and anticorrosion of robust siloxane-acrylic coatings modified with graphene nanosheets," Progress in Organic Coatings, vol. 127, pp. 239-251, 2019.

[8] S. Syed, "Influence of the environment on atmospheric corrosion of aluminium," Corrosion Engineering, Science and Technology, vol. 45, no. 4, pp. 282-287, 2013.

[9] P. A. Okafor, J. Singh-Beemat, and J. O. Iroh, "Thermomechanical and corrosion inhibition properties of graphene/epoxy ester-siloxane-urea hybrid polymer nanocomposites," Progress in Organic Coatings, vol. 88, pp. 237-244, 2015.

[10] L. Cancado, K. Takai, T. Enoki et al., "General equation for the determination of the crystallite size La of nanographite by Raman spectroscopy," Applied Physics Letters, vol. 88, no. 16, pp. 163106-163113, 2006.

[11] R. R. Laleh, H. Savaloni, F. Abdi, and Y. Abdi, "Corrosion inhibition enhancement of $\mathrm{Al}$ alloy by graphene oxide coating in $\mathrm{NaCl}$ solution," Progress in Organic Coatings, vol. 127, pp. 300-307, 2019.

[12] S. Nezamdoust and D. Seifzadeh, "rGO@APTES/hybrid solgel nanocomposite for corrosion protection of 2024 aluminum alloy," Progress in Organic Coatings, vol. 109, pp. 1-9, 2017.

[13] L. Xiong, J. Liu, M. Yu, and S. Li, "Improving the corrosion protection properties of PVB coating by using salicylaldehyde@ZIF-8/graphene oxide two-dimensional nanocomposites," Corrosion Science, vol. 146, pp. 30386-30410, 2018.

[14] S. Asaldoust and B. Ramezanzadeh, "Synthesis and characterization of a high-quality nanocontainer based on benzimidazole-zinc phosphate (ZP-BIM) tailored graphene oxides; a facile approach to fabricating a smart self-healing anti-corrosion system," Journal of Colloid and Interface Science, vol. 564, pp. 230-244, 2020.

[15] A. K. Behera, R. Chandran, S. Sarkar, and A. Mallik, "An exploration on the use of in-house synthesized reduced few layer graphene particles as a reinforcement during sonoelectroplating of $\mathrm{Cu}$ matrix composite films," Journal of Alloys and Compounds, vol. 817, p. 152713, 2020.

[16] W. Chang, P. Wang, Y. Zhao, C. Ren, B. N. Popov, and C. Li, "Characterizing corrosion properties of graphene barrier layers deposited on polycrystalline metals," Surface \& Coatings Technology, vol. 398, p. 126077, 2020.

[17] D. S. Chauhan, M. A. Quraishi, K. R. Ansari, and T. A. Saleh, "Graphene and graphene oxide as new class of materials for corrosion control and protection: Present status and future scenario," Progress in Organic Coatings, vol. 147, p. 105741, 2020.

[18] Y. Ding, J. Zhong, P. Xie et al., "Protection of Mild Steel by Waterborne Epoxy Coatings Incorporation of Nanowires/Graphene," Polymers, vol. 11, no. 12, p. 1998, 2019.

[19] S. Chhetri, S. Ghosh, P. Samanta, N. C. Murmu, and T. Kuila, "Effect of Fe3O4-decorated N-doped reduced graphene oxide nanohybrid on the anticorrosion performance of epoxy composite coating," ChemistrySelect, vol. 4, no. 46, pp. 1344613454, 2019.

[20] A. Dehghani, G. Bahlakeh, and B. Ramezanzadeh, "Designing a novel targeted-release nano-container based on the silanized graphene oxide decorated with cerium acetylacetonate loaded 
beta-cyclodextrin ( $\beta$-CD-CeA-MGO) for epoxy anticorrosion coating," Chemical Engineering Journal, vol. 400, pp. 89548-89563, 2020.

[21] L. T. Duy and H. Seo, "Construction of stretchable supercapacitors using graphene hybrid hydrogels and corrosionresistant silver nanowire current collectors," Applied Surface Science, vol. 521, p. 146467, 2020.

[22] A. A. Javidparvar, R. Naderi, and B. Ramezanzadeh, "Manipulating graphene oxide nanocontainer with benzimidazole and cerium ions: application in epoxy-based nanocomposite for active corrosion protection," Corrosion Science, vol. 165, pp. 1-61, 2020.

[23] Q. Liu, X. Zhang, W. Zhou et al., "Improved anti-corrosion behaviour of an inorganic passive film on hot-dip galvanised steel by modified graphene oxide incorporation," Corrosion Science, vol. 174, p. 108846, 2020.

[24] K. K. Pandey, A. Islam, R. Kumar, R. Ghosh, V. Arjunan, and A. K. Keshri, "Role of the hybrid addition of carbon nanotubes and graphene nanoplatelets on the corrosion behavior of plasma-sprayed aluminum oxide nanocomposite coating," Advanced Engineering Materials, vol. 22, no. 3, pp. 1-9, 2020.

[25] Q. Zhu, E. Li, X. Liu et al., "Epoxy coating with in-situ synthesis of polypyrrole functionalized graphene oxide for enhanced anticorrosive performance," Progress in Organic Coatings, vol. 140, pp. 1-23, 2020. 